\title{
Pacific
}

Journal of

Mathematics

\section{EINSTEIN METRICS AND EXOTIC SMOOTH STRUCTURES}

MASASHI ISHIDA 


\title{
EINSTEIN METRICS AND EXOTIC SMOOTH STRUCTURES
}

\author{
MASASHI ISHIDA
}

\section{We prove new existence theorems of 4-manifolds admitting infinitely many distinct smooth structures for which no Einstein metric exists.}

\section{Introduction}

A Riemannian metric $g$ is called Einstein if its Ricci curvature, considered as a function on the unit tangent bundle, is constant. It is known that any closed oriented Einstein 4-manifold $X$ satisfies

$$
2 \chi(X) \geq 3|\tau(X)|,
$$

where $\chi(X)$ and $\tau(X)$ denote respectively the Euler characteristic and signature of $X$. This is called the Hitchin-Thorpe inequality [Hitchin 1974; Thorpe 1969; Besse 1987]. Hitchin [1974] proved that any closed oriented Einstein 4-manifold satisfying $2 \chi(X)=3|\tau(X)|$ is finitely covered by either a K3 surface or the 4-torus.

On the other hand, by using Seiberg-Witten invariants [Witten 1994], LeBrun [1996] constructed the first example of a simply connected closed 4-manifold $X$ without Einstein metrics that nonetheless satisfies the strict Hitchin-Thorpe inequality $2 \chi(X)>3|\tau(X)|$. It is now well-known [LeBrun 1995a; 1995b; 2001; 2009] that the existence of monopole classes (see Definition 2 below) gives rise to obstructions to the existence of Einstein metrics on 4-manifolds. In particular, any Einstein 4-manifold $X$ with a nonzero special monopole class (see Section 2 below) must satisfy the inequality

$$
\chi(X) \geq 3 \tau(X) .
$$

This equality occurs only if $X$ is a compact quotient of the complex hyperbolic plane equipped with a constant multiple of its standard Kähler-Einstein metric. For Kähler surfaces, this inequality reduces to the celebrated Miyaoka-Yau inequality. We shall call (2) the Miyaoka-Yau-LeBrun inequality. Moreover, an obstruction found in [LeBrun 1996; 2001] provided the first means of exhibiting

This work is supported in part by the Grant-in-Aid for Scientific Research (C), Japan Society for the Promotion of Science, number 20540090.

MSC2010: primary 53C25; secondary 57R57, 57R55.

Keywords: Einstein metrics, smooth structures, Seiberg-Witten invariants. 
the dependence of Einstein metrics on smooth structures of underlying topological 4-manifolds [Kotschick 1998]. In particular, we know that there exist infinitely many topological 4-manifolds which often admit infinitely many smooth structures for which Einstein metrics do not exist, but nevertheless satisfy the Hitchin-Thorpe inequality. For instance, see [LeBrun 2001; 2003; Ishida and LeBrun 2002; 2003; Brunnbauer et al. 2009].

In this article, we shall prove the following general existence theorem of 4manifolds without Einstein metrics, which nicely highlights how much there is to be said about the subject beyond the Hitchin-Thorpe inequality (1) and the Miyaoka-Yau-LeBrun inequality (2).

Theorem A. For any pair of integers $(m, n)$ satisfying $m+n \equiv 0(\bmod 2)$, there exist infinitely many nonhomeomorphic topological nonspin 4-manifolds with

$$
(\chi, \tau)=(m, n)
$$

and all such topological nonspin 4-manifolds admit infinitely many distinct smooth structures for which nonzero special monopole classes exist and Einstein metrics do not exist. In particular:

(1) If $2 m>3|n|$, there are nonspin 4-manifolds admitting infinitely many distinct smooth structures for which no Einstein metric exists, but that nevertheless satisfy the strict Hitchin-Thorpe inequality.

(2) If $m>3 n$, there are nonspin 4-manifolds admitting infinitely many distinct smooth structures for which nonzero special monopole classes exist and Einstein metrics do not exist, but nevertheless satisfy the strict Miyaoka-YauLeBrun inequality.

Notice that any closed 4-manifold $X$ always satisfies $\chi(X)+\tau(X) \equiv 0(\bmod 2)$. Therefore, $m+n \equiv 0(\bmod 2)$ in the above theorem is the best possible. Theorem A follows from Theorem 11 proved in Section 4. Theorem 11 provides us a way to construct new examples of 4-manifolds without Einstein metrics. See also Remark 12 at the end of Section 4.

One of motivations for Theorem A comes from an interesting result due to Sambusetti [1998]. Using a remarkable inequality of Besson, Courtois, and Gallot [Besson et al. 1995] concerning the volume entropy, Sambusetti [1998] proved a topological obstruction to the existence of Einstein metrics on a 4-manifold admitting a nonzero degree map onto compact real or complex hyperbolic 4-manifolds. By applying the obstruction, Sambusetti proved an interesting existence result for 4-manifolds without Einstein metrics.

Theorem 1 [Sambusetti 1998, Theorem 4.4]. Any pair of integers $(m, n)$ satisfying $m+n \equiv 0(\bmod 2)$ can be realized as the Euler characteristic $\chi$ and signature $\tau$ 
of infinitely many nonhomeomorphic closed smooth 4-manifolds without Einstein metrics.

We notice that Sambusetti's obstruction actually depends only on the homotopy type of the manifold, and therefore in principle applies to all smooth structures. However, it appears to be unknown if most of the examples considered by Sambusetti actually admit exotic smooth structures. For instance, Theorem 1 is proved by considering the following connected sum $Y_{m, n}^{p}$ [Sambusetti 1998, Remarks 4.5]:

$$
(|m|+|n|+p) M \#(|m|+|n|-n+p) \overline{\mathbb{C} P^{2}} \#\left(|m|+|n|-\frac{m+n}{2}+1+p\right) Y,
$$

where $p$ is any nonnegative integer, $M$ is a Mumford fake projective plane [1979] and $Y:=S^{2} \times T^{2}$. We also remark that there is a vanishing theorem of Witten [1994] which asserts that all the Seiberg-Witten invariants of a connected sum $X_{1} \# X_{2}$ of 4manifolds with $b_{2}^{+}\left(X_{1}\right) \geq 1$ and $b_{2}^{+}\left(X_{2}\right) \geq 1$ vanish, where $b^{+}(X)$ is the dimension of a maximal linear subspace of $H^{2}(X, \mathbb{R})$ on which the cup product pairing is positive definite. Hence, all the Seiberg-Witten invariants of the connected sum $Y_{m, n}^{p}$ vanish in general. At least, the present author does not know how to detect the existence or nonexistence of monopole classes of $Y_{m, n}^{p}$, and, to the best of our knowledge, it is also unknown whether the underlying topological manifold of $Y_{m, n}^{p}$ admits infinitely many smooth structures for which no Einstein metric exists. Hence, Theorem A can be seen as a natural generalization of Sambusetti's result and actually contains several new aspects which were not covered by Sambusetti's result. Moreover, our method of proof is totally different from that of Theorem 1. In particular, we use the Seiberg-Witten monopole equations [Witten 1994] to prove Theorem A.

We mention that a Seiberg-Witten refinement of Theorem 1 was first proved by Del Rio Guerra [2002, Theorem D], who showed the existence of non-Einstein 4-manifolds with free fundamental group. Our Theorem A can be seen as a natural generalization of that result, because our method of proof implies the existence of topological 4-manifolds with free fundamental group and admitting infinitely many distinct smooth structures for which Einstein metrics do not exist. (See also Remark 12 below.)

Theorem A is a result on the nonspin case. The second main result of the present article tells us that a similar result still holds in the spin case:

Theorem B. For any pair of integers $(m, n)$ satisfying $m+n \equiv 0(\bmod 2)$ and $n \equiv 0(\bmod 16)$, there exist infinitely many nonhomeomorphic topological spin 4-manifolds with $(\chi, \tau)=(m, n)$ and all such topological spin 4-manifolds admit infinitely many distinct smooth structures for which nonzero special monopole classes exist and Einstein metrics do not exist. In particular: 
(1) If $2 m>3|n|$, there are spin 4-manifolds admitting infinitely many distinct smooth structures for which no Einstein metric exists, but nevertheless satisfy the strict Hitchin-Thorpe inequality.

(2) If $m>3 n$, there are spin 4-manifolds admitting infinitely many distinct smooth structures for which nonzero special monopole classes exist and Einstein metrics do not exist, but nevertheless satisfy the strict Miyaoka-Yau-LeBrun inequality.

By Rohlin's theorem, any spin 4-manifold $X$ must satisfy $\tau(X) \equiv 0(\bmod 16)$. Therefore, we cannot remove the condition $n \equiv 0(\bmod 16)$ from Theorem $\mathrm{B}$.

\section{Obstruction to the existence of Einstein metrics}

By using several nice results proved in [LeBrun 2009], we shall prove an obstruction to the existence of Einstein metrics on 4-manifold; see Theorem 7 below. We shall use the obstruction to prove the main results.

Let $X$ be a closed oriented Riemannian 4-manifold with $b^{+}(X) \geq 2$. Recall that a $\operatorname{spin}^{c}$-structure $\Gamma_{X}$ on a smooth Riemannian 4-manifold $X$ induces a pair of spinor bundles $S_{\Gamma_{X}}^{ \pm}$which are Hermitian vector bundles of rank 2 over $X$. A Riemannian metric on $X$ and a unitary connection $A$ on the determinant line bundle $\mathscr{L}_{\Gamma_{X}}:=$ $\operatorname{det}\left(S_{\Gamma_{X}}^{+}\right)$induce the twisted Dirac operator $\mathscr{D}_{A}: \Gamma\left(S_{\Gamma_{X}}^{+}\right) \rightarrow \Gamma\left(S_{\Gamma_{X}}^{-}\right)$. SeibergWitten monopole equations over $X$ are the following nonlinear partial differential equations for a unitary connection $A$ of the complex line bundle $\mathscr{L}_{\Gamma_{X}}$ and a spinor $\phi \in \Gamma\left(S_{\Gamma_{X}}^{+}\right)$:

$$
\mathscr{D}_{A} \phi=0, \quad F_{A}^{+}=i q(\phi),
$$

where $F_{A}^{+}$is the self-dual part of the curvature of $A$ and $q: S_{\Gamma_{X}}^{+} \rightarrow \wedge^{+}$is a certain natural real-quadratic map satisfying

$$
|q(\phi)|=\frac{1}{2 \sqrt{2}}|\phi|^{2},
$$

where $\wedge^{+}$is the bundle of self-dual 2-forms. We recall some background.

Definition 2 [Kronheimer 1999; Ishida and LeBrun 2003; LeBrun 2009]. Let $X$ be a closed oriented smooth 4-manifold with $b^{+}(X) \geq 2$. An element

$$
\mathfrak{a} \in H^{2}(X, \mathbb{Z}) / \text { torsion } \subset H^{2}(X, \mathbb{R})
$$

is called the monopole class of $X$ if there exists a $\operatorname{spin}^{c}$-structure $\Gamma_{X}$ with

$$
c_{1}^{\mathbb{R}}\left(\mathscr{L}_{\Gamma_{X}}\right)=\mathfrak{a},
$$


which has the property that the corresponding Seiberg-Witten monopole equations (3) have a solution for every Riemannian metric on $X$. Here $c_{1}^{\mathbb{R}}\left(\mathscr{L}_{\Gamma_{X}}\right)$ is the image of the first Chern class $c_{1}\left(\mathscr{L}_{\Gamma_{X}}\right)$ of the complex line bundle $\mathscr{L}_{\Gamma_{X}}$ in $H^{2}(X, \mathbb{R})$.

In what follows, we shall usually denote $c_{1}^{\mathbb{R}}\left(\mathscr{L}_{\Gamma_{X}}\right)$ by $c_{1}\left(\mathscr{L}_{\Gamma_{X}}\right)$ for short. A monopole class $c_{1}\left(\mathscr{L}_{\Gamma_{X}}\right)$ of $X$ is called special [Kotschick 2004] if $c_{1}^{2}\left(\mathscr{L}_{\Gamma_{X}}\right) \geq$ $2 \chi(X)+3 \tau(X)$ holds. We shall also denote the set of all monopole classes on $X$ by $\mathfrak{C}(X)$. Then we have the following fundamental result on $\mathfrak{C}(X)$.

Proposition 3 [Ishida and LeBrun 2003, Proposition 3]. Let X be a closed oriented smooth 4-manifold with $b^{+}(X) \geq 2$. Then the set $\mathfrak{C}(X)$ is a finite set.

Now recall that, for any subset $W$ of a real vector space $V$, one can consider the convex hull Hull( $W) \subset V$, meaning the smallest convex subset of $V$ containing $W$. Finiteness of $\mathfrak{C}(X)$ implies that the convex hull

$$
\operatorname{Hull}(\mathfrak{C}(X)) \subset H^{2}(X, \mathbb{R})
$$

is compact. Moreover, it is known that the convex hull $\mathrm{Hull}(\mathfrak{C}(X))$ is symmetric, that is, Hull $(\mathfrak{C}(X))=-\operatorname{Hull}(\mathfrak{C}(X))$. See [LeBrun 2009] for more details.

Since $\mathfrak{C}(X)$ is a finite set, we are able to write $\mathfrak{C}(X)=\left\{\mathfrak{a}_{1}, \mathfrak{a}_{2}, \ldots, \mathfrak{a}_{n}\right\}$. The convex hull $\operatorname{Hull}(\mathfrak{C}(X))$ is then expressed as

$$
\operatorname{Hull}(\mathfrak{C}(X))=\left\{\sum_{i=1}^{n} t_{i} \mathfrak{a}_{i} \mid t_{i} \in[0,1], \sum_{i=1}^{n} t_{i}=1\right\}
$$

Notice that the symmetric property tells us that $\operatorname{Hull}(\mathfrak{C}(X))$ contains the zero element. Let us consider the self-intersection function

$$
2: H^{2}(X, \mathbb{R}) \rightarrow \mathbb{R}
$$

which is defined by $x \mapsto x^{2}:=\langle x \cup x,[X]\rangle$, where $[X]$ is the fundamental class of $X$. Since the function 2 is a polynomial function, it is a continuous function on $H^{2}(X, \mathbb{R})$. Therefore, the restriction $\left.2\right|_{\boldsymbol{H}}$ to the compact subset $\boldsymbol{H}:=\operatorname{Hull}(\mathfrak{C}(X))$ of $H^{2}(X, \mathbb{R})$ achieves its maximum.

Definition 4 [LeBrun 2009]. Suppose $X$ is a closed oriented smooth 4-manifold with $b^{+}(X) \geq 2$. Let $\operatorname{Hull}(\mathfrak{C}(X)) \subset H^{2}(X, \mathbb{R})$ be the convex hull of the set $\mathfrak{C}(X)$ of all monopole classes on $X$. If $\mathfrak{C}(X) \neq \varnothing$, define

$$
\beta^{2}(X):=\max \left\{2(x):=x^{2} \mid x \in \operatorname{Hull}(\mathfrak{C}(X))\right\} .
$$

If instead $\mathfrak{C}(X)=\varnothing$, simply define $\beta^{2}(X):=0$.

We are now in a position to recall the following theorem. 
Theorem 5 [LeBrun 2009]. Suppose that $X$ is a closed oriented smooth 4-manifold with $b^{+}(X) \geq 2$. If $X$ admits an Einstein metric $g$,

$$
2 \chi(X)+3 \tau(X) \geq \frac{2}{3} \beta^{2}(X)
$$

with equality only if both sides vanish, in which case $g$ must be a hyper-Kähler metric, and $X$ must be diffeomorphic to $\mathrm{K} 3$ or $T^{4}$.

There are several ways to detect the existence of monopole classes. For any closed oriented smooth 4-manifold $X$ with $b^{+}(X) \geq 2$, one can define the integer valued Seiberg-Witten invariant $S W_{X}\left(\Gamma_{X}\right) \in \mathbb{Z}$ for any spin ${ }^{c}$-structure $\Gamma_{X}$ by integrating a cohomology class on the moduli space of solutions of the Seiberg-Witten monopole equations associated with $\Gamma_{X}$ :

$$
S W_{X}: \operatorname{Spin}(X) \rightarrow \mathbb{Z},
$$

where $\operatorname{Spin}(X)$ is the set of all $\operatorname{spin}^{c}$-structures on $X$. For more details, see [Witten 1994; Morgan 1996]. We call the first Chern class $c_{1}\left(\mathscr{L}_{\Gamma_{X}}\right)$ a Seiberg-Witten basic class of $X$ if $S W_{X}\left(\Gamma_{X}\right) \neq 0$ for a $\operatorname{spin}^{c}$-structure $\Gamma_{X}$. Notice that Seiberg-Witten basic classes are monopole classes.

On the other hand, there is a sophisticated refinement of the idea of the construction of the Seiberg-Witten invariant due to Bauer and Furuta [2004] (see also [Bauer 2004a; 2004b]). We call it the stable cohomotopy Seiberg-Witten invariant and denote it by $B F_{X}$. This invariant detects the presence of a monopole class via an element of a certain complicated stable cohomotopy group $\pi_{S^{1}, U}^{0}(2)$ :

$$
B F_{X}\left(\Gamma_{X}\right) \in \pi_{S^{1}, U^{(2)}}^{0} .
$$

(See [Bauer 2004a] for the construction of the stable cohomotopy group.) Under the assumption that $b^{+}(X) \geq 2$, it is also known that there is the homeomorphism

$$
t^{B F}: \pi_{S^{1}, U^{0}}^{0}(2) \rightarrow \mathbb{Z},
$$

which maps $B F_{X}\left(\Gamma_{X}\right)$ to $S W_{X}\left(\Gamma_{X}\right)$ [Bauer 2004a, Theorem 4.1 and Proposition 4.4]. In particular, this map tells us that, if $B F_{X}\left(\Gamma_{X}\right)=0$ for some $\operatorname{spin}^{c}$ structure $\Gamma_{X}$, we have $S W_{X}\left(\Gamma_{X}\right)=0$. At the same time, it is known that the nontriviality of $B F_{X}\left(\Gamma_{X}\right)$ implies that there are solutions of the following perturbed equations associated with $\Gamma_{X}$ for all metrics and all self-dual 2-forms $\eta$ :

$$
\mathscr{D}_{A} \phi=0, \quad F_{A}^{+}=i q(\phi)+i \eta .
$$

Namely, $c_{1}\left(\mathscr{L}_{\Gamma_{X}}\right)$ is a generic monopole class in the sense of [Kotschick 2004, Definition 7]. Then, by the standard argument of gauge theory, we know that $c_{1}\left(\mathscr{L}_{\Gamma_{X}}\right)$ becomes a special monopole class [Kotschick 2004, Lemma 8]. Hence 
the nontriviality of $B F_{X}\left(\Gamma_{X}\right)$ implies the existence of a special monopole class $c_{1}\left(\mathscr{L}_{\Gamma_{X}}\right)$. We shall use this fact to prove the main results.

By using $B F_{X}$, we are able to prove the following result:

Proposition 6. Let $X$ be a closed oriented smooth 4-manifold with $b^{+}(X) \geq 2$. Suppose that $S W_{X}\left(\Gamma_{X}\right) \neq 0$ holds for a spin ${ }^{c}$-structure $\Gamma_{X}$. Let $N$ be a closed oriented smooth 4-manifold with $b^{+}(N)=0$. Then a connected sum $M:=X \# N$ has monopole classes and satisfies the bound

$$
\beta^{2}(M) \geq 2 \chi(X)+3 \tau(X) .
$$

Proof. As was already mentioned, there is a comparison map (5) between $B F_{X}$ and $S W_{X}$, where we used the assumption that $b^{+}(X) \geq 2$. In particular, if $S W_{X}\left(\Gamma_{X}\right) \neq 0$ for some $\operatorname{spin}^{c}$-structure $\Gamma_{X}$, then $B F_{X}\left(\Gamma_{X}\right) \neq 0$. Then the proofs of [Ishida and LeBrun 2003, Proposition 6 and Corollary 8] (see also [Bauer 2004a, Theorem 8.8]) tell us that

$$
\pm c_{1}\left(\mathscr{L}_{\Gamma_{X}}\right)+\sum_{i=1}^{k} \pm E_{i}
$$

are monopole classes of the connected sum $M:=X \# N$, where $c_{1}\left(\mathscr{L}_{\Gamma_{X}}\right)$ is the first Chern class of the complex line bundle $\mathscr{L}_{\Gamma_{X}}$ associated with $\Gamma_{X}$. Additionally $E_{1}, E_{2}, \ldots, E_{k}$ is a set of generators for $H^{2}(N, \mathbb{Z}) /$ torsion relative to which the intersection form is diagonal and the \pm signs are arbitrary and independent of one another. In particular, by (7), we have the following two monopole classes of $M$ :

$$
\mathfrak{b}_{1}:=c_{1}\left(\mathscr{L}_{\Gamma_{X}}\right)+\sum_{i=1}^{k} E_{i}, \quad \mathfrak{b}_{2}:=c_{1}\left(\mathscr{L}_{\Gamma_{X}}\right)-\sum_{i=1}^{k} E_{i} .
$$

By (4), we obtain

$$
c_{1}(X)=\frac{1}{2} \mathfrak{b}_{1}+\frac{1}{2} \mathfrak{b}_{2} \in \operatorname{Hull}(\mathfrak{C}(M)) .
$$

We therefore get the following bound (see also Definition 4):

$$
\beta^{2}(M) \geq c_{1}^{2}\left(\mathscr{L}_{\Gamma_{X}}\right) .
$$

On the other hand, the assumption that $S W_{X}\left(\Gamma_{X}\right) \neq 0$ forces the dimension $d$ of Seiberg-Witten monopole moduli space to be nonnegative; that is,

$$
d=\frac{1}{4}\left(c_{1}^{2}\left(\mathscr{L}_{\Gamma_{X}}\right)-2 \chi(X)-3 \tau(X)\right) \geq 0 .
$$

Equivalently, we have

$$
c_{1}^{2}\left(\mathscr{L}_{\Gamma_{X}}\right) \geq 2 \chi(X)+3 \tau(X) .
$$

It is clear that (8) and (9) imply the desired bound (6). 
Theorem 5 and Proposition 6 imply the next result, a particular case of which, for $N=k \overline{\mathbb{C} P^{2}} \# \ell\left(S^{1} \times S^{3}\right)$, was proved in [LeBrun 2001, Theorem 3.3].

Theorem 7. Let $X$ be a closed oriented 4-manifold with $b^{+}(X) \geq 2$. Suppose that $S W_{X}\left(\Gamma_{X}\right) \neq 0$ holds for a spinc-structure $\Gamma_{X}$ on $X$. Let $N$ be a closed oriented smooth 4-manifold with $b^{+}(N)=0$. Then a connected sum $M:=X \# N$ cannot admit any Einstein metric if

$$
4 b_{1}(N)+b_{2}(N)>\frac{1}{3}(2 \chi(X)+3 \tau(X)) .
$$

In particular, suppose that $N$ is not an integral homology 4-sphere whose fundamental group has no nontrivial finite quotient. Then a connected sum $M:=X \# N$ cannot admit any Einstein metric if

$$
4 b_{1}(N)+b_{2}(N) \geq \frac{1}{3}(2 \chi(X)+3 \tau(X)) .
$$

Proof. Suppose that the connected sum $M:=X \# N$ admits an Einstein metric. Then Theorem 5 tells us that

$$
2 \chi(M)+3 \tau(M) \geq \frac{2}{3} \beta^{2}(M) .
$$

This bound with (6) implies

$$
2 \chi(M)+3 \tau(M) \geq \frac{2}{3}(2 \chi(X)+3 \tau(X)) .
$$

On the other hand, a direct computation tells us that

$$
2 \chi(M)+3 \tau(M)=2 \chi(X)+3 \tau(X)-\left(4 b_{1}(N)+b_{2}(N)\right) .
$$

By the bounds (12) and (13), we have

$$
2 \chi(X)+3 \tau(X)-\left(4 b_{1}(N)+b_{2}(N)\right) \geq \frac{2}{3}(2 \chi(X)+3 \tau(X)) .
$$

Equivalently,

$$
4 b_{1}(N)+b_{2}(N) \leq \frac{1}{3}(2 \chi(X)+3 \tau(X)) .
$$

By contraposition, we are able to conclude that $M$ cannot admit any Einstein metric if (10) holds.

Now suppose that $N$ is not an integral homology 4-sphere whose fundamental group has no nontrivial finite quotient. Then the equality cannot occur in (14). We shall prove this as follows. First of all, notice that Theorem 5 tells us that the equality can occur only in the case where the connected sum $M:=X \# N$ is diffeomorphic to $\mathrm{K} 3$ or $T^{4}$. Both $\mathrm{K} 3$ and $T^{4}$ are minimal Kähler surfaces. At the same time, [Kotschick 1997, Theorem 5.4] tells us that if a minimal Kähler surface with $b^{+}>1$ admits the connected sum decomposition $X \# N$, then $N$ must be an integral homology 4 -sphere whose fundamental group has no nontrivial finite 
quotient. Hence equality never occurs in (14) as desired. Therefore we conclude that, in this case, $M$ cannot admit any Einstein metric if (11) holds.

\section{Smooth structures and the geography of spin 4-manifolds}

The main result of this section is Proposition 10 below. We start by recalling a nice result of Park [2002] on the geography of spin symplectic 4-manifolds.

Let $X$ be a simply connected closed 4-manifold. We define the quantities

$$
\begin{aligned}
\chi_{h}(X) & :=\frac{\chi(X)+\tau(X)}{4}=\frac{b^{+}(X)+1}{2}, \\
c(X) & :=c_{1}^{2}(X)=2 \chi(X)+3 \tau(X)=4+5 b^{+}(X)-b^{-}(X) .
\end{aligned}
$$

Now suppose that $X$ is a spin symplectic 4-manifold with $b^{+}>1$. Then [Taubes 1994] tells us that $X$ must satisfy

$$
c(X) \geq 0 .
$$

Moreover, Rohlin's theorem forces $\tau(X) \equiv 0(\bmod 16)$. As mentioned in [Park 2002, Lemma 2.1], this is equivalent to

$$
c(X) \equiv 8 \chi_{h}(X)(\bmod 16) .
$$

The above facts tell us that the only lattice points $\left(\chi_{h}, c\right)$ satisfying both (17) and (18) can possibly be realized as $\left(\chi_{h}(X), c(X)\right)$ of a simply connected spin symplectic 4-manifold $X$. Such pairs $\left(\chi_{h}, c\right)$ of integers are called allowed lattice points.

We are now in a position to recall the following result on the geography of the spin symplectic 4-manifolds:

Theorem 8 [Park 2002, Theorem 1.1]. There is a line $c=f\left(\chi_{h}\right)$ with a slope $>8.76$ in the $\left(\chi_{h}, c\right)$-plane such that any allowed lattice point satisfying $c \leq f\left(\chi_{h}\right)$ in the first quadrant can be realized as $\left(\chi_{h}, c_{1}^{2}\right)$ of a simply connected spin noncomplex symplectic 4-manifold which admits infinitely many distinct smooth structures, all of which admit a symplectic form. In particular, all allowed lattice points $\left(\chi_{h}, c\right)$ except finitely many lying in the region $0 \leq c \leq 8.76 \chi_{h}$ satisfy $c \leq f\left(\chi_{h}\right)$.

On the other hand, let $K_{g}$ be a fibered knot in $S^{3}$ with a punctured genus $g$ surface as fiber. Let $M_{K_{g}}$ be the 3-manifold obtained by performing 0-framed surgery on $K_{g}$. Let $m$ be a meridional circle to $K_{g}$. Then the meridional circle $m$ can be seen as a circle in $M_{K_{g}}$. The 3-manifold $M_{K_{g}}$ can be consider as a fiber bundle over the circle $m$ with a closed Riemann surface $\Sigma_{g}$ as a fiber. In $M_{K_{g}} \times S^{1}$, there is a smoothly embedded torus $T_{m}=m \times S^{1}$ of self-intersection 0 . A famous result of Thurston [1976] tells us that the 4-manifold $M_{K_{g}} \times S^{1}$ admits a symplectic structure with symplectic section $T_{m}$. For any symplectic 4-manifold 
$X$ with a symplectically embedded torus $T$ of self-intersection 0 , we can consider the symplectic fiber sum $X_{M_{K g}}$ of $X$ with $M_{K_{g}} \times S^{1}$ as follows:

$$
X_{K_{g}}:=X \#_{T=T_{m}}\left(M_{K_{g}} \times S^{1}\right)=\left[X-\left(T \times D^{2}\right)\right] \cup\left[\left(M_{K_{g}} \times S^{1}\right)-\left(T_{m} \times D^{2}\right)\right],
$$

where $T \times D^{2}$ is a tubular neighborhood of the torus $T$ in the manifold $X$. Under a certain condition on $X$, Fintushel and Stern proved that $X_{K_{g}}$ is homeomorphic to $X$ and provided a way to compute the Seiberg-Witten invariants of $X_{K_{g}}$ :

Theorem 9 [Fintushel and Stern 1998, Theorem 1.5]. Let X be a simply connected symplectic 4-manifold that contains a symplectically embedded torus $T$ of selfintersection 0 in a cusp neighborhood with $\pi_{1}(X-T)=1$ and representing a nontrivial homology class [T]. If $K_{g}$ is a fibered knot, $X_{K_{g}}:=X \#_{T=T_{m}}\left(M_{K_{g}} \times S^{1}\right)$ is a symplectic 4-manifold which is homeomorphic to $X$ and whose Seiberg-Witten polynomial is given by

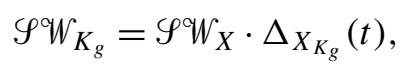

where $\Delta_{X_{K}}(t)$ is the Alexander polynomial of $K_{g}$ and $t=\exp (2[T])$.

The polynomial $\mathscr{S}^{\mathscr{Q}} W_{X}$ is defined as follows. Let $\left\{ \pm \beta_{1}, \pm \beta_{2}, \ldots, \pm \beta_{n}\right\}$ be the set of nonzero Seiberg-Witten basic classes of $X$. Then we set $b_{0}=S W_{X}(0)$, $b_{j}=S W_{X}\left(\beta_{j}\right)$, and $t_{\beta_{j}}=\exp \left(\beta_{j}\right)$. Then we define the Seiberg-Witten polynomial $\mathscr{S}^{W_{X}}$ as follows:

$$
\mathscr{S} \mathcal{W}_{X}=b_{0}+\sum_{j=1}^{n} b_{j}\left(t_{\beta_{j}}+(-1)^{(\chi(X)+\tau(X)) / 4} t_{\beta_{j}}^{-1}\right) .
$$

Since any two smooth 4-manifolds which have different Seiberg-Witten polynomials are nondiffeomorphic, one can apply Theorem 9 to construct 4-manifolds admitting infinitely many distinct smooth structures. Indeed, Park [2002] proved that every symplectic 4-manifold $W$ in Theorem 8 admits infinitely many distinct smooth structures by showing that the 4-manifold $W$ contains a symplectically embedded torus $T$ of self-intersection 0 in a cusp neighborhood with $\pi_{1}(X-T)=1$ and representing a nontrivial homology class $[T]$, that is, the 4-manifold $W$ satisfies the assumption in Theorem 9. See [Park 2002, Claim 1] for more details.

To prove the main results of the present article, we need to refine Park's result on the existence of exotic smooth structures:

Proposition 10. Let $W$ be any symplectic 4-manifold in Theorem 8 and let $N$ be any closed smooth 4-manifold with $b^{+}(N)=0$. Then the underlying topological 4-manifold of $W \# N$ admits infinitely many distinct smooth structures. 
Proof. As was already proved in [Park 2002, Claim 1], every symplectic 4-manifold $W$ in Theorem 8 contains a symplectically embedded torus $T$ of self-intersection 0 in a cusp neighborhood with $\pi_{1}(X-T)=1$ and representing a nontrivial homology class [T]. On the other hand, for any fibered knot $K_{g}$ in $S^{3}$ with a punctured genus $g$ surface as fiber, consider the 3-manifold $M_{K_{g}}$ obtained by performing 0 -framed surgery on $K_{g}$. As was already mentioned above, we are able to consider the symplectic fiber sum

$$
W_{K_{g}}=W \#_{T=T_{m}}\left(M_{K_{g}} \times S^{1}\right) .
$$

Then, Theorem 9 tells us that $W_{K_{g}}$ is homeomorphic to $W$. Now, let $N$ be any closed smooth 4-manifold with $b^{+}(N)=0$. Then we consider a connected sum $W_{K_{g}} \# N$. Of course, $W_{K_{g}} \# N$ is homeomorphic to $W \# N$. Notice that the connected sum $W_{K_{g}} \# N$ is not necessarily symplectic in general.

Next, we show that there are monopole classes of $W_{K_{g}} \# N$. In fact, [Bauer 2004a, Proposition 5.4] tells us that the comparison map (5), that is,

$$
t^{B F}: \pi_{S^{1}, U^{0}}^{0}(2) \rightarrow \mathbb{Z}
$$

becomes an isomorphism for any symplectic 4-manifold $M$ with $b^{+}(M)>1$. This fact and a result of Taubes [1994] on the nontriviality of Seiberg-Witten invariants of any symplectic 4-manifold $M$ with $b^{+}(M)>1$ imply the nontriviality of the stable cohomotopy Seiberg-Witten invariants of $M$. In particular, we can conclude that the symplectic 4-manifold $W_{K_{g}}$ has

$$
\pm c_{1}\left(\mathcal{K}_{W_{K g}}\right)
$$

as its monopole classes, where $\mathcal{K}_{W_{K g}}$ is the canonical line bundle of $W_{K_{g}}$. Since the nontriviality of the stable cohomotopy Seiberg-Witten invariants does not change under the connected sum with $N$ [Ishida and LeBrun 2003, Proposition 6], we can conclude that the connected sum $W_{K_{g}} \# N$ also has monopole classes [Ishida and LeBrun 2003, Proposition 6 and Corollary 8], that is, the cohomology classes

$$
\pm c_{1}\left(\mathscr{K}_{W_{K g}}\right)+\sum_{i=1}^{k} \pm E_{i}
$$

become monopole classes of the connected sum $W_{K_{g}} \# N$, where $E_{1}, E_{2}, \ldots, E_{k}$ is a set of generators for $H^{2}(N, \mathbb{Z})$ /torsion relative to which the intersection form is diagonal and the \pm signs are arbitrary and independent of one another.

On the other hand, following the argument at the beginning of the proof of [Fintushel and Stern 1998, Corollary 1.7], we are able to express $c_{1}\left(\mathscr{K}_{W_{K_{g}}}\right)$ more explicitly. For the reader, let us explain it here. First of all, notice that the homology $H_{2}\left(M_{K_{g}} \times S^{1}\right)$ is generated by the classes of the symplectic curves $T_{m}$ and $\Sigma_{g}$. This 
tells us that the first Chern class $c_{1}\left(\mathscr{K}_{M_{K g} \times S^{1}}\right)$ of the canonical line bundle $\mathscr{K}_{M_{K g} \times S^{1}}$ of the symplectic 4-manifold $M_{K_{g}} \times S^{1}$ has the form

$$
c_{1}\left(\mathcal{K}_{K_{g} \times S^{1}}\right)=\alpha\left[T_{m}\right]+\beta\left[\Sigma_{g}\right] .
$$

Since we have $\left[T_{m}\right]^{2}=\left[\Sigma_{g}\right]^{2}=0$ and $\left[T_{m}\right] \cdot\left[\Sigma_{g}\right]=1$,

$$
c_{1}\left(\mathscr{K}_{K_{g} \times S^{1}}\right) \cdot\left[\Sigma_{g}\right]=\alpha, \quad c_{1}\left(\mathscr{K}_{W_{K g}}\right) \cdot\left[T_{m}\right]=\beta .
$$

These facts and the adjunction formula tell us that $\alpha=2 g-2$ and $\beta=0$ hold. Therefore, we conclude that

$$
c_{1}\left(\mathscr{K}_{K_{g} \times S^{1}}\right)=(2 g-2)\left[T_{m}\right]=(2 g-2)[T] .
$$

On the other hand, $c_{1}\left(\mathscr{K}_{W_{K g}}\right)=c_{1}\left(\mathscr{K}_{W}\right)+c_{1}\left(\mathcal{K}_{K_{g} \times S^{1}}\right)+2[T]$ holds by the construction of $W_{K_{g}}$. Therefore,

$$
c_{1}\left(\mathscr{K}_{W_{K_{g}}}\right)=c_{1}\left(\mathscr{K}_{W}\right)+c_{1}\left(\mathscr{K}_{K_{g} \times S^{1}}\right)+2[T]=c_{1}\left(\mathscr{K}_{W}\right)+2 g[T] .
$$

By (19) and (20), we conclude that

are monopole classes of $W_{K_{g}} \# N$.

$$
\pm\left(c_{1}\left(\mathscr{K}_{W}\right)+2 g[T]\right)+\sum_{i=1}^{k} \pm E_{i}
$$

By considering an infinite sequence $\left\{K_{g_{\ell}}\right\}_{\ell \in \mathbb{N}}$ of fibered knots with $g_{\ell} \geq 1$, where the genus $g_{\ell}$ is strictly increasing with respect to $\ell$, that is, $g_{\ell} \rightarrow \infty$ when $\ell \rightarrow \infty$, we have an infinite sequence $\left\{W_{K_{g_{\ell}}} \# N\right\}_{\ell \in \mathbb{N}}$ of smooth 4-manifolds which are homeomorphic to $W \# N$ and, for each $\ell$,

$$
\pm\left(c_{1}\left(\mathscr{K}_{W}\right)+2 g_{\ell}[T]\right)+\sum_{i=1}^{k} \pm E_{i}
$$

are monopole classes of $W_{K_{g \ell}} \# N$. Suppose now that the sequence

$$
\left\{W_{K_{g_{\ell}}} \# N\right\}_{\ell \in \mathbb{N}}
$$

contains only finitely many diffeomorphism types. Specifically, suppose that there exists a positive integer $\ell_{0}$ such that $W_{K_{g \ell_{0}}} \# N$ is diffeomorphic to $W_{K_{g \ell_{i}}} \# N$ for any $\ell_{i} \geq \ell_{0}$. Then, by the expression (21) of the monopole classes and taking $\ell_{i} \rightarrow \infty$, we conclude that the set of monopole classes of 4-manifold $W_{K_{g_{\ell_{0}}}} \# N$ is unbounded. However, this is a contradiction because the set of monopole classes of any given smooth 4-manifold with $b^{+}>1$ must be finite by Proposition 3. Therefore, the sequence $\left\{W_{K_{g \ell}} \# N\right\}_{\ell \in \mathbb{N}}$ actually contains infinitely many diffeomorphism types. As was already mentioned above, since each 4-manifold $W_{K_{g_{\ell}}} \# N$ is homeomorphic to $W \# N$, the underlying topological 4-manifold of $W \# N$ admits infinitely many distinct smooth structures as desired. 


\section{Proof of Theorem A}

Theorem 11. Let $Z$ be a closed oriented smooth 4-manifold satisfying $b^{+}(Z)=0$, $b_{1}(Z) \neq 0$, and

$$
0<\frac{b_{2}(Z)}{b_{1}(Z)}<\frac{19}{50}
$$

where $b_{1}(Z)$ and $b_{2}(Z)$ denote the first and second Betti numbers of $Z$, respectively. Let $M$ be any closed oriented smooth 4-manifold with $b_{1}(M)=0, b^{+}(M)=$ 0 , and $b_{2}(M)=1$.

For such 4-manifolds as $Z$ and $M$, and for any pair of integers $(m, n)$ satisfying $m+n \equiv 0(\bmod 2)$, there exist infinitely many nonhomeomorphic topological nonspin 4-manifolds $X_{Z, M}^{m, n}$ with $(\chi, \tau)=(m, n)$, and all such topological nonspin 4-manifolds admit infinitely many distinct smooth structures for which nonzero special monopole classes exist and Einstein metrics do not exist.

Proof. For any pair of integers $(m, n)$ satisfying $m+n \equiv 0(\bmod 2)$, it is easy to see that there exist infinitely many pairs $(k, \ell)$ of sufficiently large positive integers satisfying the following three conditions:

$$
(x, y):=\left(\frac{m+n+2 b_{1}(Z) \ell}{4}, 2 m+3 n+k+\left(4 b_{1}(Z)+b_{2}(Z)\right) \ell\right) \in \mathscr{D},
$$

$$
\begin{gathered}
n+k+b_{2}(Z) \ell \equiv 0(\bmod 16), \\
k+\left(4 b_{1}(Z)+b_{2}(Z)\right) \ell>\frac{1}{3} y,
\end{gathered}
$$

where $\mathscr{D}$ is the set of all pairs of integers satisfying the conditions of Theorem 8 . Notice that the condition (23) is equivalent to

$$
y \equiv 8 x(\bmod 16) .
$$

Moreover, the condition (22) was already used, that is,

$$
8.76>\frac{\left(4 b_{1}(Z)+b_{2}(Z)\right) \ell}{\left(2 b_{1}(Z) \ell\right) / 4}=8+2 \frac{b_{2}(Z)}{b_{1}(Z)},
$$

or, equivalently,

$$
\frac{b_{2}(Z)}{b_{1}(Z)}<0.38=\frac{19}{50} .
$$

By Theorem 8 , for each $(x, y)$ above, there is a simply connected spin noncomplex symplectic 4-manifold $W$ with

$$
\begin{aligned}
\chi_{h}(W) & :=\frac{\chi(W)+\tau(W)}{4}=x=\frac{m+n+2 b_{1}(Z) \ell}{4}, \\
c_{1}^{2}(W) & :=2 \chi(W)+3 \tau(W)=y=2 m+3 n+k+\left(4 b_{1}(Z)+b_{2}(Z)\right) \ell .
\end{aligned}
$$


Hence we obtain

$$
\begin{aligned}
m+n & =\chi(W)+\tau(W)-2 b_{1}(Z) \ell \\
2 m+3 n & =2 \chi(W)+3 \tau(W)-k-\left(4 b_{1}(Z)+b_{2}(Z)\right) \ell .
\end{aligned}
$$

Consider the connected sum

$$
X_{Z, M}^{m, n}=W \# M \#(k-1) \overline{\mathbb{C} P^{2}} \# \ell Z .
$$

Notice that $X_{Z, M}^{m, n}$ is nonspin. On the other hand, by a direct computation, we obtain

$$
\chi\left(M \#(k-1) \overline{\mathbb{C} P^{2}} \# \ell Z\right)=k+\chi(\ell Z)=k+2+b_{2}(Z) \ell-2 b_{1}(Z) \ell .
$$

Notice that $\chi(M)=3$ because we assume that $b_{1}(M)=0, b^{+}(M)=0$, and $b_{2}(M)=1$. Therefore we get

$$
\chi\left(X_{Z, M}^{m, n}\right)=\chi(W)+\chi\left(M \#(k-1) \overline{\mathbb{C} P^{2}}\right)-2=\chi(W)+k+b_{2}(Z) \ell-2 b_{1}(Z) \ell .
$$

Similarly, we have

$$
\begin{aligned}
\tau\left(M \#(k-1) \overline{\mathbb{C} P^{2}} \# \ell Z\right) & =\tau(M)+\tau\left((k-1) \overline{\mathbb{C} P^{2}}\right)+\tau(\ell Z) \\
& =-1+(1-k)-b_{2}(Z) \ell \\
& =-k-b_{2}(Z) \ell .
\end{aligned}
$$

Notice that $b^{+}(Z)=0$, so we have $\tau(Z)=-b_{2}(Z)$. Therefore

$$
\tau\left(X_{Z, M}^{m, n}\right)=\tau(W)+\tau\left(M \#(k-1) \overline{\mathbb{C} P^{2}} \# \ell Z\right)=\tau(W)-k-b_{2}(Z) \ell .
$$

By (29) and (30), we get

$$
\begin{aligned}
\chi\left(X_{Z, M}^{m, n}\right)+\tau\left(X_{Z, M}^{m, n}\right) & =\chi(W)+\tau(W)-2 b_{1}(Z) \ell, \\
2 \chi\left(X_{Z, M}^{m, n}\right)+3 \tau\left(X_{Z, M}^{m, n}\right) & =2 \chi(W)+3 \tau(W)-k-\left(4 b_{1}(Z)+b_{2}(Z)\right) \ell .
\end{aligned}
$$

Then (26), (27), (31), and (32) immediately tell us that

$$
\chi\left(X_{Z, M}^{m, n}\right)=m, \quad \tau\left(X_{Z, M}^{m, n}\right)=n
$$

On the other hand, by $b_{1}(W)=b_{1}(M)=b_{1}\left(\overline{\mathbb{C} P^{2}}\right)=0$, we have

$$
b_{1}\left(X_{Z, M}^{m, n}\right)=b_{1}(Z) \ell .
$$

Since there are infinitely many choices of $\ell,(33)$ and (34) tell us that, for $(m, n)$ satisfying $m+n \equiv 0(\bmod 2)$, there exist infinitely many nonhomeomorphic nonspin 4-manifolds $X_{Z, M}^{m, n}$ with $(\chi, \tau)=(m, n)$. Now set $N:=M \#(k-1) \overline{\mathbb{C} P^{2}} \# \ell Z$. We write $X_{Z, M}^{m, n}=W \# N$. Notice that $N$ satisfies $b^{+}(N)=0$.

By considering an infinite sequence $\left\{K_{g_{i}}\right\}_{i \in \mathbb{N}}$ of fibered knots with $g_{i} \geq 1$ as the proof of Proposition 10 above, we obtain the sequence $\left\{W_{K_{g_{i}}} \# N\right\}_{i \in \mathbb{N}}$ which 
contains infinitely many diffeomorphism types by Proposition 10, and every 4manifold $X_{g_{i}}:=W_{K_{g_{i}}} \# N$ is homeomorphic to $X_{Z, M}^{m, n}=W \# N$. To prove that the 4-manifold $X_{Z, M}^{m, n}$ admits infinitely many distinct smooth structures for which nonzero monopole classes exist and Einstein metrics do not exist, it is enough to prove that the smooth 4-manifold $X_{g_{i}}$ has nonzero special monopole classes and cannot admit any Einstein metric. It is clear that $X_{g_{i}}$ has nonzero special monopole classes by the proof of Proposition 10, where the nontriviality of $B F$ implies the existence of a special monopole class. On the other hand, since any symplectic 4-manifold with $b^{+}>1$ has nontrivial Seiberg-Witten invariants by a result of Taubes [1994] and $N$ satisfies $b^{+}(N)=0$, Theorem 7 tells us that if

$$
k+\left(4 b_{1}(Z)+b_{2}(Z)\right) \ell>\frac{1}{3}\left(2 \chi\left(W_{K_{g_{i}}}\right)+3 \tau\left(W_{K_{g_{i}}}\right)\right),
$$

the manifold $X_{g_{i}}:=W_{K_{g_{i}}} \# N$ cannot admit any Einstein metric. By Park's construction of $W$ and Theorem $9, W_{K_{g_{i}}}$ is homeomorphic to $W$ and we therefore have

$$
2 \chi\left(W_{K_{g_{i}}}\right)+3 \tau\left(W_{K_{g_{i}}}\right)=2 \chi(W)+3 \tau(W) .
$$

So the bound (35) is equivalent to

$$
k+\left(4 b_{1}(Z)+b_{2}(Z)\right) \ell>\frac{1}{3}(2 \chi(W)+3 \tau(W)) .
$$

However, the bound (36) automatically holds because we have (24) and (25). Therefore, $X_{g_{i}}:=W_{K_{g_{i}}} \# N$ cannot admit any Einstein metric as desired.

Theorem A immediately follows from Theorem 11. Indeed, it is enough to find a smooth closed 4-manifold satisfying (22) and a closed oriented smooth 4-manifold $M$ with $b_{1}(M)=0, b^{+}(M)=0$, and $b_{2}(M)=1$. For example, set $M:=\overline{\mathbb{C} P^{2}}$ and $Z:=11 K \# 4 \overline{\mathbb{C} P^{2}}$, where $K$ is a secondary Kodaira surface; cf. [Barth et al. 1984]. We have $b_{1}(K)=1, b^{+}(K)=0$, and $b_{2}(K)=0$. It is clear that $M$ satisfies $b_{1}(M)=0, b^{+}(M)=0$, and $b_{2}(M)=1$. We also have $b_{1}(Z)=11, b_{2}^{+}(Z)=0$, and $b_{2}(Z)=4$. Therefore, we get

$$
\frac{b_{2}(Z)}{b_{1}(Z)}=\frac{4}{11}<\frac{19}{50} \text {. }
$$

Hence $Z:=11 K \# 4 \overline{\mathbb{C} P^{2}}$ is a 4-manifold satisfying (22). Hence we have proved Theorem A by considering the connected sum

$$
X_{11 K \# 4 \overline{\mathbb{C} P^{2}}}^{m, n}, \overline{\mathbb{C} P^{2}}=W \# k \overline{\mathbb{C} P^{2}} \# \ell\left(11 K \# 4 \overline{\mathbb{C} P^{2}}\right) ;
$$

see (28).

Remark 12. We are able to use another negative definite 4-manifold satisfying (22) to prove Theorem A. For example, we are able to use another connected sum 
$a T \# b \bar{Y}$, or $a T \# b \overline{\mathbb{C} P^{2}}$ as $Z$ above by taking a suitable pair of positive integers $(a, b)$ for which the condition (22) is satisfied. Here $\bar{Y}$ is a Mumford fake projective plane [Mumford 1979] with the reversed orientation, and $T$ is $S^{1} \times S^{3}$, or a secondary Kodaira surface. If we take $Z$ as $a\left(S^{1} \times S^{3}\right) \# b \overline{\mathbb{C} P^{2}}$, then the resulting 4-manifold $X_{Z, \bar{C} P^{2}}^{m, n}$ has a free fundamental group. See also [Del Rio Guerra 2002].

\section{Proof of Theorem B}

A method similar to that used in the proof of Theorem 11 enables us to prove Theorem B:

Theorem 13. For any pair of integers $(m, n)$ satisfying $m+n \equiv 0(\bmod 2)$ and $n \equiv 0(\bmod 16)$, there exist infinitely many nonhomeomorphic topological spin 4-manifolds with $(\chi, \tau)=(m, n)$ and all such topological spin 4-manifolds admit infinitely many distinct smooth structures for which nonzero special monopole classes exist and Einstein metrics do not exist.

Proof.

For any pair of integers $(m, n)$ satisfying $m+n \equiv 0(\bmod 2)$ and $n \equiv 0(\bmod 16)$, we are able to see that there exist infinitely many, sufficiently large positive integers $\ell$ satisfying

$$
\begin{aligned}
(x, y): & =\left(\frac{m+n+2 \ell}{4}, 2 m+3 n+4 \ell\right) \in \mathscr{D}, \\
4 \ell & >\frac{1}{3} y,
\end{aligned}
$$

where $\mathscr{D}$ is the set of all pairs of integers satisfying the conditions of Theorem 8 . In particular, notice that $y \equiv 8 x(\bmod 16)$ must be satisfied, that is,

$$
2 m+3 n+4 \ell \equiv 8\left(\frac{m+n+2 \ell}{4}\right)(\bmod 16) .
$$

Specifically, we have

$$
2 m+3 n+4 \ell \equiv 2 m+2 n+4 \ell(\bmod 16) .
$$

This is nothing but $n \equiv 0(\bmod 16)$. By Theorem 8 , for each $(x, y)$ above, there is a simply connected spin noncomplex symplectic 4-manifold $W$ with

$$
\begin{aligned}
& \chi_{h}(W):=\frac{\chi(W)+\tau(W)}{4}=x=\frac{m+n+2 \ell}{4}, \\
& c_{1}^{2}(W):=2 \chi(W)+3 \tau(W)=y=2 m+3 n+4 \ell .
\end{aligned}
$$

We obtain

$$
\begin{aligned}
m+n & =\chi(W)+\tau(W)-2 \ell, \\
2 m+3 n & =2 \chi(W)+3 \tau(W)-4 \ell .
\end{aligned}
$$


Let us consider the connected sum

$$
X^{m, n}=W \# \ell\left(S^{1} \times S^{3}\right) .
$$

Notice that $X^{m, n}$ is spin. We also get

$$
\begin{aligned}
\chi\left(X^{m, n}\right)+\tau\left(X^{m, n}\right) & =\chi(W)+\tau(W)-2 \ell, \\
2 \chi\left(X^{m, n}\right)+3 \tau\left(X^{m, n}\right) & =2 \chi(W)+3 \tau(W)-4 \ell .
\end{aligned}
$$

By (39), (40), (41), and (42), we obtain

$$
\chi\left(X^{m, n}\right)=m, \quad \tau\left(X^{m, n}\right)=n .
$$

On the other hand, we have $b_{1}(W)=0$ and $b_{1}\left(S^{1} \times S^{3}\right)=1$. Therefore, we get

$$
b_{1}\left(X^{m, n}\right)=\ell .
$$

Since there are infinitely many choices of $\ell,(43)$ and (44) implies that, for $(m, n)$ satisfying $m+n \equiv 0(\bmod 2)$ and $n \equiv 0(\bmod 16)$, there exist infinitely many nonhomeomorphic spin 4-manifolds $X^{m, n}$ with $(\chi, \tau)=(m, n)$.

We set $N:=\ell\left(S^{1} \times S^{3}\right)$ and write $X^{m, n}=W \# N$. Notice that $N$ satisfies $b^{+}(N)=0$. By considering an infinite sequence $\left\{K_{g_{i}}\right\}_{i \in \mathbb{N}}$ of fibered knots with $g_{i} \geq 1$ as proof of Theorem 11, we obtain the sequence $\left\{W_{K_{g_{i}}} \# N\right\}_{i \in \mathbb{N}}$ which contains infinitely many diffeomorphism types. Every 4-manifold $X_{g_{i}}:=W_{K_{g_{i}}} \# N$ is homeomorphic to $X^{m, n}=W \# N$ and has nonzero special monopole classes. Moreover, $X_{g_{i}}$ cannot admit any Einstein metric as follows. By Theorem 7, if

$$
4 \ell>\frac{1}{3}\left(2 \chi\left(W_{K_{g_{i}}}\right)+3 \tau\left(W_{K_{g_{i}}}\right)\right),
$$

the manifold $X_{g_{i}}:=W_{K_{g_{i}}} \# N$ cannot admit any Einstein metric. Since

$$
2 \chi\left(W_{K_{g_{i}}}\right)+3 \tau\left(W_{K_{g_{i}}}\right)=2 \chi(W)+3 \tau(W),
$$

the bound (45) is equal to

$$
4 \ell>\frac{1}{3}(2 \chi(W)+3 \tau(W)) .
$$

However, the bound (46) automatically holds because of (37) and (38). Therefore, we conclude that $X_{g_{i}}:=W_{K_{g_{i}}} \# N$ cannot admit any Einstein metric as desired.

\section{Remarks on the simply connected case}

NonEinstein 4-manifolds constructed in Theorem A, Theorem B, and Theorem 1 are not simply connected. It is natural to ask whether one can prove simply connected versions of these theorems. This is an open problem, and is closely related to the following. 
Question 14 [LeBrun 2001, Question 3.5]. For every $q \in(-1,1) \cap \mathbb{Q}$, are there smooth, compact simply connected 4-manifolds with $\tau / \chi=q$ which do not admit Einstein metrics?

LeBrun [2001] gives a partial affirmative answer to this question under $0.351 \leq$ $|q|<1$ [LeBrun 2001, Corollary 3.6]. However, a complete solution to this question is still unknown.

On the other hand, in the present article, we have seen that 4-manifolds often admit infinitely many distinct smooth structures for which no Einstein metric exists. In light of this phenomenon, we would like to consider a generalization of Question 14:

Question 15. For every $q \in(-1,1) \cap \mathbb{Q}$, are there compact simply connected topological 4-manifolds with $\tau / \chi=q$ which admit infinitely many distinct smooth structures for which no Einstein metrics exist?

To prove a result in this direction, we need to recall the following:

Theorem 16 [Park 2003, Theorem 1.1]. There is an increasing sequence $\left\{m_{i}\right\}$ with $m_{i} \rightarrow 9$ such that every simply connected closed, nonspin, irreducible smooth 4manifold $X$ satisfying $0 \leq c(X) \leq m_{i} \chi_{h}(X)$ and $b^{+}(X) \geq C_{i}$, where $C_{i}$ is an odd constant depending on $m_{i}$, admits infinitely many, both symplectic and nonsymplectic, exotic smooth structures.

Applying the idea of the proof of [LeBrun 2001, Corollary 3.6] and the preceding result, we obtain:

Corollary 17. For any rational number $q \in \mathbb{Q}$ satisfying

$$
\frac{1}{3}<|q|<1,
$$

there exist compact simply connected topological 4-manifolds with $\tau / \chi=q$ admitting infinitely many distinct smooth structures for which no Einstein metric exists.

Proof. We will actually prove that there is an increasing sequence $\left\{n_{i}\right\}$ such that $n_{i} \rightarrow-1 / 3$ satisfying the following property: For any rational number $q$ with

$$
-1<q \leq n_{i},
$$

there exist compact simply connected topological 4-manifolds with $\tau / \chi=q$ admitting infinitely many distinct smooth structures for which no Einstein metrics exists.

Let $\alpha$ and $\beta$ be any positive integers satisfying

$$
\frac{s}{t} \in\left(0, m_{i}\right]
$$

where $m_{i}$ is an increasing sequence with $m_{i} \rightarrow 9$ in Theorem 16 . Then, Theorem 16 specifically tells us that, for any sufficiently large integers $\ell$, there is a simply 
connected nonspin 4-manifold $X$ admitting infinitely many distinct symplectic structures and with

$$
\begin{aligned}
& \chi_{h}(X)=\frac{\chi(X)+\tau(X)}{4}=t \ell, \\
& c_{1}^{2}(X)=2 \chi(X)+3 \tau(X)=s \ell .
\end{aligned}
$$

We denote the infinite family of symplectic 4-manifolds which are homeomorphic to $X$ by $\left\{Y_{n}\right\}$. For each symplectic 4-manifold $Y_{n}$ which is homeomorphic to $X$, consider the $k$-times blow-up $M_{n}^{k}:=Y_{n} \# k \overline{\mathbb{C} P^{2}}$ of $Y_{n}$ where $k$ satisfies

$$
k \geq \frac{1}{3}\left(2 \chi\left(Y_{n}\right)+3 \tau\left(Y_{n}\right)\right)=\frac{1}{3}(2 \chi(X)+3 \tau(X)) .
$$

By (48), this is equivalent to

$$
\frac{k}{s \ell} \geq \frac{1}{3}
$$

Theorem 7 tells us that $M_{n}^{k}$ cannot admit any Einstein metric. Moreover, for each $k$, the infinite family $\left\{M_{n}^{k}\right\}$ of symplectic 4-manifolds also contains infinitely many diffeomorphism types because the difference of smooth structures survives under blow-ups. This means that, for each $k$, the underlying topological 4-manifold of $X_{k}:=X \# k \overline{\mathbb{C} P^{2}}$ admits infinitely many smooth structures $\left\{M_{n}^{k}\right\}$ without Einstein metrics.

On the other hand, we have $\chi_{h}\left(M_{n}^{k}\right)=\chi_{h}(X)$ and $c_{1}^{2}\left(M_{n}^{k}\right)=c_{1}^{2}(X)-k$ because $Y_{n}$ is homeomorphic to $X$. Using this fact, (47), and (48), we have

$$
\frac{c_{1}^{2}\left(M_{n}^{k}\right)}{\chi_{h}\left(M_{n}^{k}\right)}=\frac{c_{1}^{2}(X)-k}{\chi_{h}(X)}=\frac{s \ell-k}{t \ell}=\frac{s}{t}\left(1-\frac{k}{s \ell}\right) .
$$

Since $k / s \ell \in[1 / 3, \infty) \cap \mathbb{Q}$, we get

$$
1-\frac{k}{s \ell} \in\left(-\infty, \frac{2}{3}\right] \cap \mathbb{Q} \text {. }
$$

Since we also have $s / t \in\left(0, m_{i}\right]$, the following holds.

$$
\frac{c_{1}^{2}\left(M_{n}^{k}\right)}{\chi_{h}\left(M_{n}^{k}\right)} \in\left(-\infty, \frac{2}{3} m_{i}\right] \cap \mathbb{Q} .
$$

On the other hand, as was already mentioned in the proof of [LeBrun 2001, Corollary 3.6], we get

$$
\frac{\tau\left(M_{n}^{k}\right)}{\chi\left(M_{n}^{k}\right)}=\left(3-\frac{1}{4} \frac{c_{1}^{2}\left(M_{n}^{k}\right)}{\chi_{h}\left(M_{n}^{k}\right)}\right)^{-1}-1 .
$$

By (49), we have

$$
\left(3-\frac{1}{4} \frac{c_{1}^{2}\left(M_{n}^{k}\right)}{\chi_{h}\left(M_{n}^{k}\right)}\right)^{-1} \in\left(0, \frac{6}{18-m_{i}}\right] \cap \mathbb{Q} .
$$


This tells us that

$$
\frac{\tau\left(M_{n}^{k}\right)}{\chi\left(M_{n}^{k}\right)} \in\left(-1,-1+\frac{6}{18-m_{i}}\right] \cap \mathbb{Q} .
$$

Since the sequence $m_{i} \rightarrow 9$ is increasing, we have an increasing sequence $\left\{n_{i}\right\}$ such that

$$
n_{i} \rightarrow-\frac{1}{3}
$$

by setting

$$
n_{i}:=-1+\frac{6}{18-m_{i}} .
$$

Since we have $\tau\left(M_{n}^{k}\right)=\tau\left(X_{k}\right)$ and $\chi\left(M_{n}^{k}\right)=\tau\left(X_{k}\right)$, the above tells us that, for any rational number $q$ with

$$
-1<q \leq n_{i}
$$

there exist compact simply connected topological 4-manifolds $X_{k}$ with $\tau / \chi=q$ admitting infinitely many distinct smooth structures $\left\{M_{n}^{k}\right\}$ for which no Einstein metrics exists. The case where $q$ is positive then follows by reversing the orientation of the above examples.

\section{Acknowledgement}

The author thanks the referee for valuable comments and suggestions.

\section{References}

[Barth et al. 1984] W. Barth, C. Peters, and A. Van de Ven, Compact complex surfaces, Ergebnisse der Mathematik und ihrer Grenzgebiete (3) [Results in Mathematics and Related Areas (3)] 4, Springer, Berlin, 1984. MR 86c:32026 Zbl 0718.14023

[Bauer 2004a] S. Bauer, "Refined Seiberg-Witten invariants", pp. 1-46 in Different faces of geometry, edited by S. Donaldson et al., Int. Math. Ser. (N. Y.) 3, Kluwer/Plenum, New York, 2004. MR 2005h:57045 Zbl 1083.57039

[Bauer 2004b] S. Bauer, "A stable cohomotopy refinement of Seiberg-Witten invariants. II", Invent. Math. 155:1 (2004), 21-40. MR 2005c:57041 Zbl 1051.57038

[Bauer and Furuta 2004] S. Bauer and M. Furuta, "A stable cohomotopy refinement of SeibergWitten invariants. I", Invent. Math. 155:1 (2004), 1-19. MR 2005c:57040 Zbl 1050.57024

[Besse 1987] A. L. Besse, Einstein manifolds, Ergebnisse der Mathematik und ihrer Grenzgebiete (3) [Results in Mathematics and Related Areas (3)] 10, Springer, Berlin, 1987. MR 88f:53087 Zbl 0613.53001

[Besson et al. 1995] G. Besson, G. Courtois, and S. Gallot, "Entropies et rigidités des espaces localement symétriques de courbure strictement négative”, Geom. Funct. Anal. 5:5 (1995), 731-799. MR 96i:58136 Zbl 1194.01076

[Brunnbauer et al. 2009] M. Brunnbauer, M. Ishida, and P. Suárez-Serrato, "An essential relation between Einstein metrics, volume entropy, and exotic smooth structures", Math. Res. Lett. 16:3 (2009), 503-514. MR 2011g:53088 Zbl 1183.53038 
[Del Rio Guerra 2002] H. Del Rio Guerra, "Seiberg-Witten invariants of nonsimple type and Einstein metrics”, Ann. Global Anal. Geom. 21:4 (2002), 319-339. MR 2003g:53062 Zbl 1011.53038

[Fintushel and Stern 1998] R. Fintushel and R. J. Stern, "Knots, links, and 4-manifolds", Invent. Math. 134:2 (1998), 363-400. MR 99j:57033 Zbl 0914.57015

[Hitchin 1974] N. Hitchin, "Compact four-dimensional Einstein manifolds", J. Differential Geometry 9 (1974), 435-441. MR 50 \#3149 Zbl 0281.53039

[Ishida and LeBrun 2002] M. Ishida and C. LeBrun, "Spin manifolds, Einstein metrics, and differential topology”, Math. Res. Lett. 9:2-3 (2002), 229-240. MR 2003f:53079 Zbl 1017.53045

[Ishida and LeBrun 2003] M. Ishida and C. LeBrun, "Curvature, connected sums, and SeibergWitten theory”, Comm. Anal. Geom. 11:5 (2003), 809-836. MR 2005c:53042 Zbl 1084.58501

[Kotschick 1997] D. Kotschick, "The Seiberg-Witten invariants of symplectic four-manifolds (after C. H. Taubes)", pp. 195-220, exposé No. 812, 4, in Séminaire Bourbaki, Vol. 1995/96, Astérisque 241, Soc. Math. de France, Paris, 1997. MR 98h:57057 Zbl 0882.57026

[Kotschick 1998] D. Kotschick, "Einstein metrics and smooth structures", Geom. Topol. 2 (1998), 1-10. MR 99h:57060 Zbl 0885.57014

[Kotschick 2004] D. Kotschick, "Monopole classes and Einstein metrics", Int. Math. Res. Not. 2004:12 (2004), 593-609. MR 2004k:53062 Zbl 1051.57038

[Kronheimer 1999] P. B. Kronheimer, "Minimal genus in $S^{1} \times M^{3}$ ”, Invent. Math. 135:1 (1999), 45-61. MR 2000c:57071 Zbl 0917.57017

[LeBrun 1995a] C. LeBrun, "Einstein metrics and Mostow rigidity", Math. Res. Lett. 2:1 (1995), 1-8. MR 95m:53067 Zbl 0974.53035

[LeBrun 1995b] C. LeBrun, "Polarized 4-manifolds, extremal Kähler metrics, and Seiberg-Witten theory”, Math. Res. Lett. 2:5 (1995), 653-662. MR 96h:58038 Zbl 0874.53051

[LeBrun 1996] C. LeBrun, "Four-manifolds without Einstein metrics", Math. Res. Lett. 3:2 (1996), 133-147. MR 97a:53072 Zbl 0856.53035

[LeBrun 2001] C. LeBrun, "Ricci curvature, minimal volumes, and Seiberg-Witten theory", Invent. Math. 145:2 (2001), 279-316. MR 2002h:53061 Zbl 0999.53027

[LeBrun 2003] C. LeBrun, "Einstein metrics, four-manifolds, and differential topology", pp. 235255 in Surveys in differential geometry (Boston, MA, 2002), edited by S.-T. Yau, Surv. Differ. Geom. 8, Int. Press, Somerville, MA, 2003. MR 2005g:53078 Zbl 1051.53038

[LeBrun 2009] C. LeBrun, "Four-manifolds, curvature bounds, and convex geometry", pp. 119-152 in Riemannian topology and geometric structures on manifolds, edited by K. Galicki and S. R. Simanca, Progr. Math. 271, Birkhäuser, Boston, MA, 2009. MR 2010h:53045 Zbl 1191.57023

[Morgan 1996] J. W. Morgan, The Seiberg-Witten equations and applications to the topology of smooth four-manifolds, Mathematical Notes 44, Princeton University Press, 1996. MR 97d:57042 Zbl 0846.57001

[Mumford 1979] D. Mumford, "An algebraic surface with $K$ ample, $\left(K^{2}\right)=9, p_{g}=q=0$ ", Amer. J. Math. 101:1 (1979), 233-244. MR 80j:14032 Zbl 0433.14021

[Park 2002] J. Park, "The geography of Spin symplectic 4-manifolds", Math. Z. 240:2 (2002), 405421. MR 2003c:57030 Zbl 1030.57032

[Park 2003] J. Park, "Exotic smooth structures on 4-manifolds. II", Topology Appl. 132:2 (2003), 195-202. MR 2004d:57033 Zbl 1028.57032

[Sambusetti 1998] A. Sambusetti, "An obstruction to the existence of Einstein metrics on 4-manifolds”, Math. Ann. 311:3 (1998), 533-547. MR 99k:53101 Zbl 0920.53026 
[Taubes 1994] C. H. Taubes, "The Seiberg-Witten invariants and symplectic forms", Math. Res. Lett. 1:6 (1994), 809-822. MR 95j:57039 Zbl 0853.57019

[Thorpe 1969] J. A. Thorpe, "Some remarks on the Gauss-Bonnet integral", J. Math. Mech. 18 (1969), 779-786. MR 41 \#963 Zbl 0183.50503

[Thurston 1976] W. P. Thurston, "Some simple examples of symplectic manifolds", Proc. Amer. Math. Soc. 55:2 (1976), 467-468. MR 53 \#6578 Zbl 0324.53031

[Witten 1994] E. Witten, "Monopoles and four-manifolds", Math. Res. Lett. 1:6 (1994), 769-796. MR 96d:57035 Zbl 0856.53035

Received October 31, 2010. Revised May 16, 2012.

MASASHI ISHIDA

DEPARTMENT OF MATHEMATICS

SOPHIA UNIVERSITY

7-1 KIOI-CHO

CHIYODA-KU, TOKYO 102-8554

JAPAN

ishida-m@hoffman.cc.sophia.ac.jp 


\title{
PACIFIC JOURNAL OF MATHEMATICS
}

\author{
http://pacificmath.org \\ Founded in 1951 by \\ E. F. Beckenbach (1906-1982) and F. Wolf (1904-1989)
}

\section{EDITORS}

V. S. Varadarajan (Managing Editor)

Department of Mathematics

University of California

Los Angeles, CA 90095-1555

pacific@math.ucla.edu

Vyjayanthi Chari

Department of Mathematics

University of California

Riverside, CA 92521-0135

chari@math.ucr.edu

\section{Robert Finn}

Department of Mathematics Stanford University

Stanford, CA 94305-2125

finn@math.stanford.edu

Kefeng Liu

Department of Mathematics

University of California

Los Angeles, CA 90095-1555

liu@math.ucla.edu
Darren Long

Department of Mathematics

University of California

Santa Barbara, CA 93106-3080

long@math.ucsb.edu

Jiang-Hua Lu

Department of Mathematics

The University of Hong Kong

Pokfulam Rd., Hong Kong jhlu@maths.hku.hk

Alexander Merkurjev

Department of Mathematics

University of California

Los Angeles, CA 90095-1555

merkurev@math.ucla.edu
Sorin Popa

Department of Mathematics University of California

Los Angeles, CA 90095-1555 popa@math.ucla.edu

Jie Qing

Department of Mathematics

University of California

Santa Cruz, CA 95064

qing@cats.ucsc.edu

Jonathan Rogawski

Department of Mathematics

University of California

Los Angeles, CA 90095-1555

jonr@math.ucla.edu

\section{PRODUCTION}

pacific@math.berkeley.edu

\section{SUPPORTING INSTITUTIONS}

ACADEMIA SINICA, TAIPEI

CALIFORNIA INST. OF TECHNOLOGY INST. DE MATEMÁTICA PURA E APLICADA KEIO UNIVERSITY

MATH. SCIENCES RESEARCH INSTITUTE NEW MEXICO STATE UNIV.

OREGON STATE UNIV.

\author{
STANFORD UNIVERSITY \\ UNIV. OF BRITISH COLUMBIA \\ UNIV. OF CALIFORNIA, BERKELEY \\ UNIV. OF CALIFORNIA, DAVIS \\ UNIV. OF CALIFORNIA, LOS ANGELES \\ UNIV. OF CALIFORNIA, RIVERSIDE \\ UNIV. OF CALIFORNIA, SAN DIEGO \\ UNIV. OF CALIF., SANTA BARBARA
}

\author{
UNIV. OF CALIF., SANTA CRUZ \\ UNIV. OF MONTANA \\ UNIV. OF OREGON \\ UNIV. OF SOUTHERN CALIFORNIA \\ UNIV. OF UTAH \\ UNIV. OF WASHINGTON \\ WASHINGTON STATE UNIVERSITY
}

These supporting institutions contribute to the cost of publication of this Journal, but they are not owners or publishers and have no responsibility for its contents or policies.

See inside back cover or pacificmath.org for submission instructions.

The subscription price for 2012 is US \$420/year for the electronic version, and \$485/year for print and electronic.

Subscriptions, requests for back issues from the last three years and changes of subscribers address should be sent to Pacific Journal of Mathematics, P.O. Box 4163, Berkeley, CA 94704-0163, U.S.A. Prior back issues are obtainable from Periodicals Service Company, 11 Main Street, Germantown, NY 12526-5635. The Pacific Journal of Mathematics is indexed by Mathematical Reviews, Zentralblatt MATH, PASCAL CNRS Index, Referativnyi Zhurnal, Current Mathematical Publications and the Science Citation Index.

The Pacific Journal of Mathematics (ISSN 0030-8730) at the University of California, c/o Department of Mathematics, 969 Evans Hall, Berkeley, CA 94720-3840, is published monthly except July and August. Periodical rate postage paid at Berkeley, CA 94704, and additional mailing offices. POSTMASTER: send address changes to Pacific Journal of Mathematics, P.O. Box 4163, Berkeley, CA 94704-0163.

PJM peer review and production are managed by EditFLOW ${ }^{\mathrm{TM}}$ from Mathematical Sciences Publishers.

PUBLISHED BY PACIFIC JOURNAL OF MATHEMATICS

at the University of California, Berkeley 94720-3840

A NON-PROFIT CORPORATION

Typeset in LATEX

Copyright $(02012$ by Pacific Journal of Mathematics 


\section{PACIFIC JOURNAL OF MATHEMATICS}

Volume $258 \quad$ No. $2 \quad$ August 2012

Uniqueness theorems for $\mathrm{CR}$ and conformal mappings

YounG-JUn CHOI and JAE-CHEON JOO

Some finite properties for vertex operator superalgebras

269

CHONGYING DONG and JIANZHI HAN

On the geometric flows solving Kählerian inverse $\sigma_{k}$ equations

291

HAO FANG and MIJIA LAI

An optimal anisotropic Poincaré inequality for convex domains

GUOFANG WANG and CHAO XIA

Einstein metrics and exotic smooth structures

MASASHI ISHIDA

Noether's problem for $\hat{S}_{4}$ and $\hat{S}_{5}$

MING-CHANG KANG and JiAn ZHOU

Remarks on the behavior of nonparametric capillary surfaces at corners 369

KIRK E. LANCASTER

Generalized normal rulings and invariants of Legendrian solid torus links

MIKHAIL LAVROV and DAN RUTHERFORD

Classification of singular $\mathbb{Q}$-homology planes II: $\mathbb{C}^{1}$ - and $\mathbb{C}^{*}$-rulings.

KAROL PALKA

A dynamical interpretation of the profile curve of CMC twizzler surfaces

OSCAR M. PERDOMO

Classification of Ising vectors in the vertex operator algebra $V_{L}^{+}$

HIROKI SHIMAKURA

Highest-weight vectors for the adjoint action of $\mathrm{GL}_{n}$ on polynomials 